\title{
THE CONTINUED OPTICAL TO MID-INFRARED EVOLUTION OF V838 MONOCEROTIS*
}

\author{
S. R. Loebman ${ }^{1,13}$, J. P. Wisniewski ${ }^{2,14}$, S. J. Schmidt ${ }^{3}$, A. F. KowAlski ${ }^{4}$, R. K. BARRY ${ }^{5}$, K. S. Bjorkman ${ }^{6,14}$, H. B. Hammel ${ }^{7}$, \\ S. L. Hawley ${ }^{8}$, L. HebB ${ }^{9}$, M. M. Kasliwal ${ }^{10,15}$, D. K. LyNCH ${ }^{11,14}$, R. W. Russell ${ }^{11,14}$, M. L. SitKo ${ }^{12,14,16}$, AND P. SzKODY ${ }^{8}$ \\ ${ }^{1}$ Department of Astronomy, University of Michigan, 830 Dennison, 500 Church Street, Ann Arbor, MI 48109-1042, USA; sloebman@umich.edu \\ ${ }^{2}$ Homer L. Dodge Department of Physics \& Astronomy, The University of Oklahoma, 440 W. Brooks Street, Norman, OK 73019, USA \\ ${ }^{3}$ Department of Astronomy, Ohio State University, 140 West 18th Avenue, Columbus, OH 43210, USA \\ ${ }^{4}$ NASA Postdoctoral Program Fellow, NASA Goddard Space Flight Center, Code 671, Greenbelt, MD 20771, USA \\ 5 NASA Goddard Space Flight Center, Laboratory for Exoplanets \& Stellar Astrophysics, Code 667, Greenbelt, MD 20771, USA \\ ${ }^{6}$ Ritter Observatory, MS \#113, Department of Physics \& Astronomy, University of Toledo, Toledo, OH 43606-3390, USA \\ ${ }^{7}$ AURA, 1212 New York Avenue NW, Suite 450, Washington, DC 20005, USA \\ ${ }^{8}$ Department of Astronomy, University of Washington, Box 351580, Seattle, WA 98195, USA \\ ${ }^{9}$ Department of Physics, Hobart \& William Smith Colleges, 300 Pulteney Street, Geneva, NY 14456, USA \\ ${ }^{10}$ The Observatories, Carnegie Institution for Science, 813 Santa Barbara Street, Pasadena, CA 91101, USA \\ ${ }^{11}$ The Aerospace Corporation, M2-266, P.O. Box 92957, Los Angeles, CA 90009-29257, USA \\ ${ }^{12}$ Department of Physics, University of Cincinnati, Cincinnati OH 45221, USA \\ Received 2014 June 19; accepted 2014 September 7; published 2014 December 15
}

\begin{abstract}
The eruptive variable V838 Monocerotis (V838 Mon) gained notoriety in 2002 when it brightened nine magnitudes in a series of three outbursts and then rapidly evolved into an extremely cool supergiant. We present optical, near-infrared (near-IR), and mid-IR spectroscopic and photometric observations of V838 Mon obtained between 2008 and 2012 at the Apache Point Observatory $3.5 \mathrm{~m}$, NASA IRTF $3 \mathrm{~m}$, and Gemini South $8 \mathrm{~m}$ telescopes. We contemporaneously analyze the optical and IR spectroscopic properties of V838 Mon to arrive at a revised spectral type L3 supergiant and effective temperature $T_{\text {eff }} \sim 2000-2200 \mathrm{~K}$. Because there are no existing optical observational data for L supergiants, we speculate that V838 Mon may represent the prototype for $\mathrm{L}$ supergiants in this wavelength regime. We find a low level of $\mathrm{H} \alpha$ emission present in the system, consistent with interaction between V838 Mon and its B3V binary; however, we cannot rule out a stellar collision as the genesis event, which could result in the observed $\mathrm{H} \alpha$ activity. Based upon a two-component blackbody fit to all wavelengths of our data, we conclude that, as of 2009, a shell of ejecta surrounded V838 Mon at a radius of $R=263 \pm 10 \mathrm{AU}$ with a temperature of $T=285 \pm 2 \mathrm{~K}$. This result is consistent with IR interferometric observations from the same era and predictions from the Lynch et al. model of the expanding system, which provides a simple framework for understanding this complicated system.
\end{abstract}

Key words: circumstellar matter - stars: general - stars: individual (V838 Monocerotis) - stars: peculiar - stars: variables: general - stars: winds, outflows

Supporting material: data behind figures

\section{INTRODUCTION}

V838 Monocerotis (henceforth V838 Mon) is a dramatic stellar object: in 2002, it brightened nine magnitudes during three outbursts (Brown et al. 2002; Goranskii et al. 2002), experienced a variety of distinctive optical and infrared (IR) changes (Wisniewski et al. 2003; Lynch et al. 2004; Rushton et al. 2005; Geballe et al. 2007), and eventually morphed into a L-type supergiant (Evans et al. 2003). V838 Mon is estimated to be located at a distance of $6.2 \pm 1.2 \mathrm{kpc}$ (Sparks et al. 2008) and is embedded in a sparse, young cluster with an upper age limit of $\sim 25 \mathrm{Myr}$ and an intervening reddening $E(B-V)=0.85 \quad$ (Afşar \& Bond 2007; Tylenda \& Kamiński 2012). Since its outburst, an unresolved B3V

\footnotetext{
* This publication is partially based on observations obtained with the Apache Point Observatory $3.5 \mathrm{~m}$ telescope, which is owned and operated by the Astrophysical Research Consortium.

13 Postdoctoral Fellow, Michigan Society of Fellows.

${ }^{14}$ Visiting Astronomer at the Infrared Telescope Facility, which is operated by the University of Hawaii under Cooperative Agreement no. NNX-08AE38A with the National Aeronautics and Space Administration, Science Mission Directorate, Planetary Astronomy Program.

${ }^{15}$ Hubble Fellow.

16 Also at Space Science Institute, 4750 Walnut Avenue, Suite 205, Boulder, CO 80301, USA.
}

companion in the system has been detected through spectroscopic monitoring (Munari et al. 2005).

Many theoretical mechanisms have been explored to explain the origin of V838 Mon's outbursts (Lawlor 2005; Munari et al. 2005; Retter et al. 2006; Tylenda \& Soker 2006); the most likely scenario is a stellar merger between two progenitor stars in a formerly triple system (Tylenda \& Soker 2006). This scenerio also predicts X-ray emission from the spin-up of the envelope produced by such a merger (Soker \& Tylenda 2007); however, such X-ray emission has not been seen in two epochs of Chandra imagery (Antonini et al. 2010). The light from V838 Mon's B3V companion is sufficient to account for the entire luminosity of the variable star measured on sky-survey photographs before its outburst (Afşar \& Bond 2007; however, see Barsukova et al. 2010 for a discussion of the progenitor as its own B3V star).

While V838 Mon is a decidedly rare object, several analogs have been reported in the literature. Two emerging classes of cool explosions are: luminous red novae (LRNe) and intermediate luminosity red transients (ILRTs). Akin to V838 Mon, LRNe are stellar eruptions that have remained extremely cool through the outburst and include V1309 Sco (Tylenda et al. 2011) and V4332 Sgr (Martini et al. 1999). Two extragalactic events have also been noted to be LRNe: one in 
the bulge of M31 (Mould et al. 1990) and one in the lenticular galaxy M85 (Kulkarni et al. 2007). It has been proposed that LRNe are stellar mergers. ILRTs are similarly red but too luminous to be explained by stellar mergers: e.g., SN 2008S in NGC 6946 (Prieto et al. 2008), NGC 300-OT 2008 (Bond et al. 2009; Thompson et al. 2009), and PTF 10fqs in M99 (Kasliwal et al. 2011). Consistent with their discovery in grand spirals, it has been proposed that ILRTs represent electroncapture induced collapse in extreme asymptotic giant branch stars (Kochanek 2011).

The local circumstellar environment of V838 Mon has been studied extensively since the initial outburst of the system. Based on an observed, short-lived (2002 FebruaryMarch) intrinsic polarization in the system, the outburst geometry was likely non-spherical (Wisniewski et al. 2003). In 2002 October, a temporary re-emergence of an intrinsic polarization component occurred. Oriented $90^{\circ}$ from the original component, the new polarization suggests one of two changes to the system: either a change in the optical depth of the material surrounding the $\operatorname{star}(\mathrm{s})$ or a physical shift in the illuminating source(s) (Wisniewski et al. 2003). Recent interferometric observations (Chesneau et al. 2014) have indicated a similar local circumstellar geometry as suggested by polarimetric data. V838 Mon is also surrounded by a substantially broader region of nebular material as diagnosed by the dramatic light echo illuminated by the outburst events (Bond et al. 2003).

Continued observations of V838 Mon's local circumstellar environment have traced the dynamical evolution of the system. Optical and IR photometric monitoring has suggested that the outburst ejecta have advanced past the location of the B3V binary, completely attenuating its signal (Goranskij et al. 2008) and condensing to form new circumstellar dust (Wisniewski et al. 2008). To explain the evolution of spectroscopic and photometric properties observed over time, Lynch et al. (2004) proposed a simple, spherically symmetric model containing a central star with an expanding circumstellar shell that is cooling in a radiatively dominated quasiequilibrium manner; this model is consistent with spectral fits to optical and IR data from Lynch et al. (2004). More recently, Lynch et al. (2007) expanded this conceptual framework to include five components: a central star, two photospheric shells that surround the central star, and two much cooler and more distant expanding shells of gas. Validating the central tenents of these models, including the number of components required and their expansion velocities, has remained elusive due to the previously limited number of epochs (2) of IR data available.

In this paper, we discuss the continued evolution of the system in the context of the Lynch et al. (2004, 2007) models and previously published photometric, spectroscopic, and spectro-polarimetric observations of the system. In Section 2, we present optical, near-IR, and mid-IR spectroscopic and mid-IR photometric observations of V838 Mon obtained between 2008 and 2012 at the Apache Point Observatory (APO) 3.5 m, NASA Infrared Telescope Facility (IRTF) 3 m, and Gemini South $8 \mathrm{~m}$ telescopes. In Section 3, we show that a low level of $\mathrm{H} \alpha$ emission has returned to the system, possibly due to excitation of the gas by the B3V companion as seen through the optically thinning gas and dust ejecta or due to magnetic activity produced by an emerging dynamo effect from the proposed stellar merger. In Section 4, we
Table 1

Summary of Optical and IR Spectroscopic and Photometric Data

\begin{tabular}{|c|c|c|c|}
\hline Date & Observatory & Instrument & Wavelength Coverage/Filters \\
\hline 2008 Oct 12 & APO & ARCES & $3600-10000 \AA$ \\
\hline 2009 Nov 01 & $\cdots$ & $\cdots$ & $\cdots$ \\
\hline 2009 Nov 27 & $\ldots$ & $\ldots$ & $\ldots$ \\
\hline 2010 Feb 27 & $\cdots$ & $\ldots$ & $\cdots$ \\
\hline 2010 Mar 25 & $\cdots$ & $\cdots$ & $\cdots$ \\
\hline 2012 Sep 21 & $\cdots$ & $\cdots$ & $\cdots$ \\
\hline 2008 Oct 12 & APO & DIS & blue: $4500-5500 \AA$ \\
\hline 2008 Nov 23 & $\cdots$ & $\cdots$ & red: $6000-9000 \AA$ \\
\hline 2009 Jan 15 & $\cdots$ & $\cdots$ & $\cdots$ \\
\hline 2009 Apr 19 & Gemini South & T-ReCS & N Lo-Res: 7.7-12.97 $\mu \mathrm{m}$ \\
\hline \multirow[t]{3}{*}{2009 Sep 20} & $\ldots$ & $\ldots$ & Si-5: $11.09-12.22 \mu \mathrm{m}$ \\
\hline & $\cdots$ & $\cdots$ & Si-6: $11.74-12.92 \mu \mathrm{m}$ \\
\hline & $\cdots$ & $\cdots$ & Qa: $17.57-19.08 \mu \mathrm{m}$ \\
\hline 2008 Nov 26 & NASA IRTF & SpeX & $0.8-2.5 \mu \mathrm{m}$ \\
\hline 2009 Jan 10 & $\cdots$ & $\cdots$ & . \\
\hline 2008 Sep 05 & NASA IRTF & BASS & $2.9-13.5 \mu \mathrm{m}$ \\
\hline 2009 Dec 02 & $\cdots$ & $\cdots$ & $\cdots$ \\
\hline
\end{tabular}

spectrally type V838 Mon in both the optical (M7 supergiant) and near-IR (L3 supergiant) and discuss the discrepancy in the two identifications. In Section 5, we fit our 2009 observations to the Lynch et al. (2004) model for the expanding warm dust envelope and find our data is consistent with the previous two epochs of data. Finally, in Section 6, we discuss the implications of the results we presented in the previous sections.

\section{OBSERVATIONS}

Optical spectra were taken using the Astrophysical Research Consortium Echelle Spectrograph (ARCES) and the Dual Imaging Spectrograph (DIS) instruments on APO $3.5 \mathrm{~m}$ telescope between 2008-2012 (see Table 1). ARCES (Wang et al. 2003) is a high resolution, cross-dispersed visible light spectrograph $^{17}$ that obtains spectra between 3600-10000 with a resolution of $R \sim 31500$. Standard bias, flat field, and ThAr lamp exposures for the echelle were also obtained on every night. These data were reduced using standard techniques in IRAF. ${ }^{18}$

DIS is a medium dispersion spectrograph with separate red and blue channels. ${ }^{19}$ Our DIS observations were taken using the default low-resolution blue and red gratings (B400/R300), yielding a resolution $R \sim 800$ and a total effective wavelength coverage of 4500-9000 ̊. Bias, flat field, and HeNeAr lamp exposures for DIS were obtained on every night, and nightly observations of the flux standard stars G191B2b, Feige34, or Feige 110 were used to flux calibrate these data. These data were reduced using standard techniques in IRAF.

We obtained two epochs of observations of V838 Mon (Table 1) using SpeX (Rayner et al. 2003), a mediumresolution $0.8-5.4 \mu \mathrm{m}$ spectrograph located at NASA's IRTF. ${ }^{20}$ We used the $0.3 \times 15^{\prime \prime}$. 0 slit for our V838 Mon observations, providing $R \sim 2000$ spectra from $0.8-2.5 \mu \mathrm{m}$, and also

\footnotetext{
17 www.apo.nmsu.edu/arc35m/Instruments/ARCES/

18 IRAF is distributed by the National Optical Astronomy Observatories, which are operated by the Association of Universities for Research in Astronomy, Inc., under cooperative agreement with the National Science Foundation.

19 www.apo.nmsu.edu/arc35m/Instruments/DIS/

${ }^{20}$ http://irtfweb.ifa.hawaii.edu/ spex/
} 


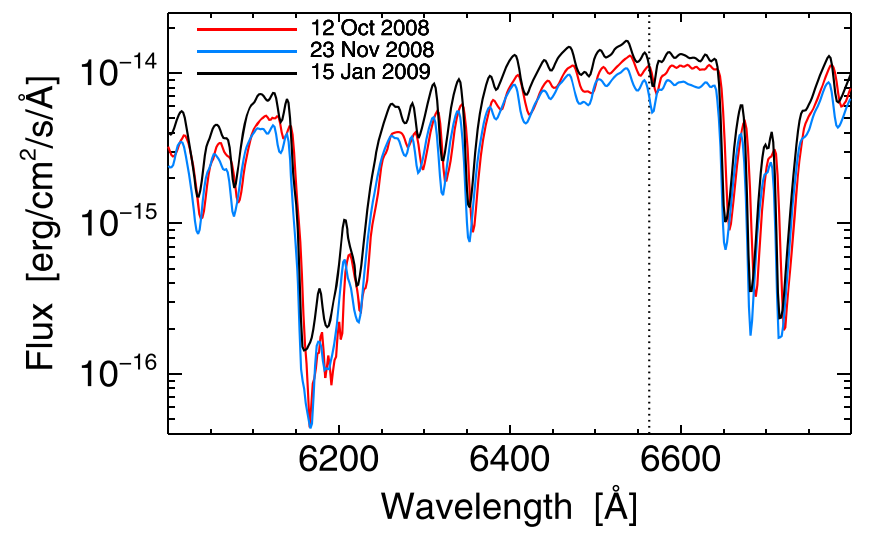

Figure 1. We monitored the optical spectrum of V838 Mon at low resolution $(R \sim 800)$ with the DIS instrument on the $3.5 \mathrm{~m}$ Apache Point Observatory telescope from 2008-2009. We find no convincing evidence of emission at this resolution, consistent with that noted by Bond (2009). The data used to create this figure are available.

obtained observations of the A0V star HD 53205 to provide telluric correction. These data were reduced using Spextool (Vacca et al. 2003; Cushing et al. 2004).

Mid-IR low resolution spectroscopy and imaging of V838 Mon were obtained with Thermal-Region Camera Spectrograph (T-ReCS), located at the Gemini South Observatory. ${ }^{21}$ As summarized in Table 1 , we obtained both low resolution $(R \sim 100)$ spectroscopy in the $N$-band, narrowband photometry near the $N$-band $(\mathrm{Si}-5: 11.7 \mu \mathrm{m}$ and $\mathrm{Si}-6$ : $12.3 \mu \mathrm{m})$, and broadband photomtry in the $Q$-band (Qa: $18.3 \mu \mathrm{m})$. Standard telescope chopping and nodding was used to mitigate the effects of the thermal background of the sky in the mid-IR. These data were reduced using the midir Gemini/ IRAF software package.

We also present two epochs of mid-IR photometry of V838 Mon obtained with the Broadband Array Spectrograph System (BASS), mounted on NASA's IRTF (see Table 1). BASS is an IR array prism spectrograph (Hackwell et al. 1990) that covers the 2.9-13.5 $\mu \mathrm{m}$ spectral region simultaneously at a resolving power of 25-120, depending on wavelength, and records these data on 116 back-illuminated blocked impurity band Si: As detectors. ${ }^{22}$ Our BASS observations were calibrated using $\alpha$ Tau as a reference star. We note that relative calibrations for reference stars $\alpha$ Tau, $\alpha \mathrm{CMa}, \beta$ Gem, $\alpha$ Boo, and $\alpha$ Lyr have remained constant to about $1 \%$ with repeated tests.

\section{3. $\mathrm{H} \alpha$ EVOLUTION}

The $\mathrm{H} \alpha$ line has proven to be an important diagnostic of the evolution of V838 Mon's circumstellar environment, helping to trace the initial expansion of ejecta from the 2002 outbursts (Wisniewski et al. 2003) and in 2006 probing the likely interaction of this expanding ejecta with the B3V companion (Munari et al. 2007). We discuss the continued monitoring of $\mathrm{H} \alpha$ between 2008 October and 2012 September below.

In Figure 1, we present three optical spectra of V838 Mon taken with the low resolution $(R \sim 800)$ DIS spectrograph on APO. We detect no convincing evidence of

\footnotetext{
21 www.gemini.edu/sciops/instruments/trecs/

22 www.aero.org/capabilities/remotesensing/bass.html
}

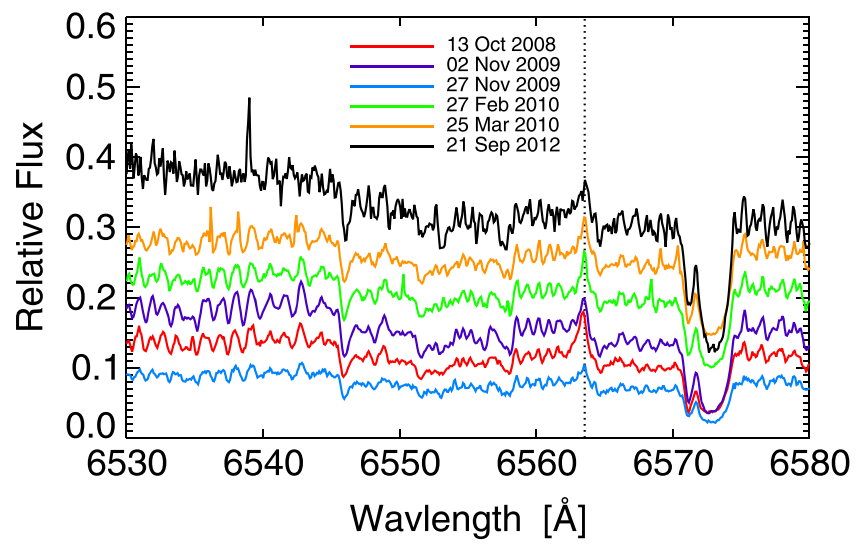

Figure 2. We also monitored V838 Mon using the high resolution $(R \sim 31500)$ Echelle spectrograph on the $3.5 \mathrm{~m}$ Apache Point Observatory telescope. At this resolution, we see clear evidence of a low level of H $\alpha$ emission from 2008 October-2012 September. Note, we have added a fiducial offset to each spectrum for ease of comparison. The data used to create this figure are available.

$\mathrm{H} \alpha$ emission at this resolution, which is consistent with conclusions drawn by Bond (2009). However, the three optical spectra of V838 Mon taken with the high resolution $(R \sim 31500)$ Echelle spectrograph on APO during the same epoch as our low resolution spectra clearly reveal the presence of a low level of $\mathrm{H} \alpha$ emission (Figure 2). This low level of emission still appears present during our fourth epoch of high resolution spectra obtained in 2012. We do not observe any significant variability in the $\mathrm{H} \alpha$ emission. These results are consistent with the report of low level $\mathrm{H} \alpha$ emission being present in 2009-epoch high resolution spectra by Tylenda et al. (2011), and extend the time period over which the system exhibits this emission.

One possible origin for this low level emission could be excitation of the expanding dust and gas envelope by the B3V companion. Bond (2009) suggests that the behavior of $\mathrm{H} \alpha$ from 200 to 2009, which included a brief return of the line appearing in emission followed by a return to an apparent pure absorption profile, was due to the $\mathrm{B} 3 \mathrm{~V}$ companion becoming completely engulfed by the expanding envelope. However, our high resolution spectra suggest that perhaps this envelope is not completely optically thick. Alternatively, the observed low level $\mathrm{H} \alpha$ emission could be caused by magnetic activity in the primary star, which is an expected byproduct of the proposed stellar merger event of 2002 (Soker \& Tylenda 2007). We discuss the plausibility of each of these scenarios in Section 6.

\section{SPECTRAL TYPING}

To better understand the properties of the central star, we compare both the DIS optical spectrum and SpeX IR spectrum with other cool objects. For the purposes of this discussion, we assume that the dust shell has little or no effect on the spectral features, and have corrected for a reddening of $E(B-V)=0.85$.

\subsection{Optical Spectral Type}

For a starting point in our analysis, we compare the optical spectrum of V838 Mon to M5-M9 dwarf (Bochanski et al. 2007) and giant (Pickles 1998) spectroscopic templates 


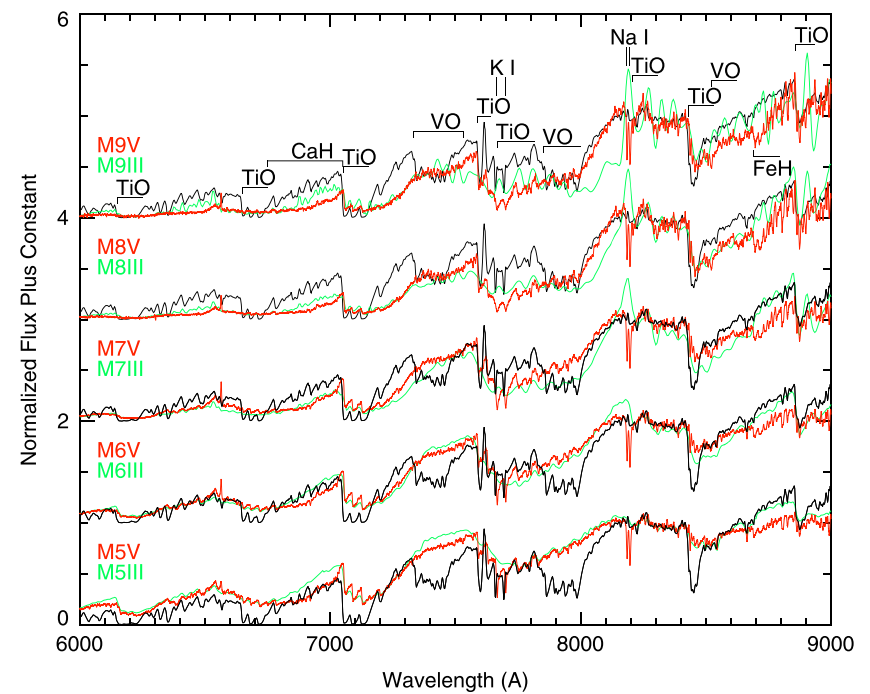

Figure 3. Comparison of our low-resolution $(R \sim 800)$, extinction corrected $(E(B-V)=0.85)$ optical spectrum of V838 Mon (black) with M dwarf templates (red) from Bochanski et al. (2007) and M giant templates (green) from Pickles (1998). Prominent molecular and atomic features are labeled. Due to the dramatic TiO bands, the best match spectra are M7. We note that the optical spectrum of V838 Mon shows narrower molecular bands and atomic lines than both the dwarfs and giants due to its low surface gravity.

(shown in Figure 3). We used the Hammer spectral typing software (Covey et al. 2007) to estimate the optical spectral type based on the characteristics of $\mathrm{M}$ dwarf optical spectra. We obtain a best fit type of M7, consistent with the Tylenda et al. (2011) type of M6.3. An examination of the specific optical features, however, indicates that the type is primarily based on the strength of the TiO bands, and most optical features are more sensitive to the V838 Mon's surface gravity rather than its surface temperature.

The optical spectrum of V838 Mon shows strong, sharp TiO and VO features, with only weak $\mathrm{CaH}$ absorption at $6750 \AA$ and no sign of $\mathrm{FeH}$ absorption at $8600 \AA$. The presence and strength of $\mathrm{TiO}$ and $\mathrm{VO}$ is consistent with an oxygen-rich stellar atmosphere, and the absence of $\mathrm{FeH}$ and $\mathrm{CaH}$ bands indicate a very low gravity atmosphere; those bands are weak or absent in M giants (Evans et al. 2003). The sharpness of the $\mathrm{TiO}$ and VO bands and the $\mathrm{K}$ I doublet at $7700 \AA$ compared to both the dwarf and giant templates indicates that it is likely to have an even lower surface gravity (consistent with a supergiant classification). The TiO bands at $6150 \AA, 6651 \AA$, and $7053 \AA$ each absorb to zero flux, indicating the absence of the B3 component detected by Munari et al. (2005).

There is no detection of the $8183 / 8195 \AA \mathrm{Na}$ I doublet, which is typically in absorption for late-M dwarfs and in emission for late-M giants (Schiavon et al. 1997). It is possible that the lack of emission is due to the lower gravity of V838 Mon compared to $\mathrm{M}$ giants. The absence of this feature is perhaps not remarkable due to the weak or absent $\mathrm{Na}$ I absorption both in the optical (Tylenda et al. 2011; the $\sim 5893 \AA$ doublet is very weak in the 2009 spectrum) and the near-IR (we do not detect the $\mathrm{Na}$ I doublets at $\sim 1.14$ and $\sim 2.21 \mu \mathrm{m}$; see Section 4.2).

\subsection{Infrared Spectral Type}

Evans et al. (2003) noted that the 0.8-2.4 $\mu \mathrm{m}$ spectrum of V838 Mon appeared to be that of an L supergiant star. There
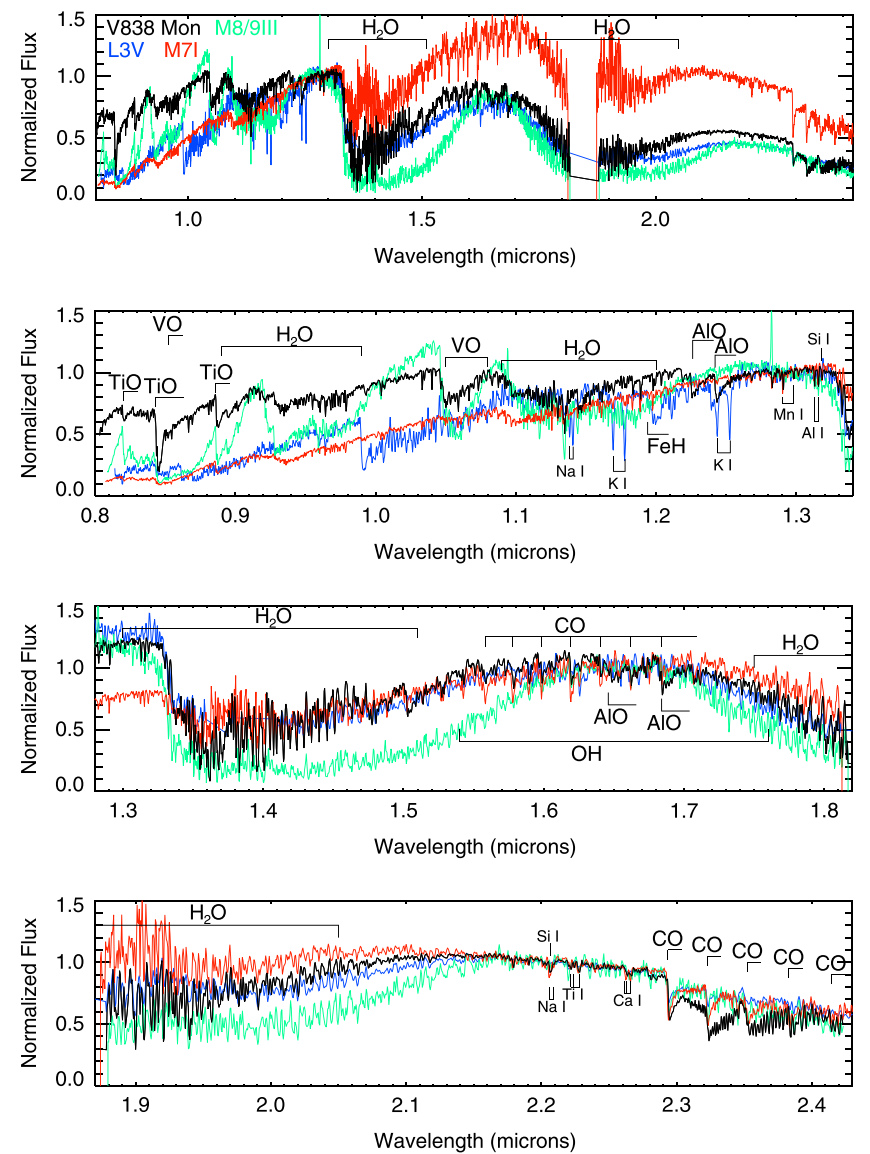

Figure 4. Comparison of our IRTF SpeX data (black lines) to spectra from the IRTF Spectral Library (colored lines; Cushing et al. 2005; Rayner et al. 2009). The top panel shows the entire range of the SpeX data, while the next three panels show the $J_{-}, H$-, and $K$-band respectively. The types of each spectrum are given in the top left corner of the top panel. None of the comparison spectra are a perfect match for the V838 Mon spectrum. The $\mathrm{L} 3 \mathrm{~V}$ is the best match for the overall spectral slope, while the M8/9III is the best match in the $J$-band due to the dramatic TiO bands. The M7I spectrum is the best match in the $H$ - and $K$-bands. The data used to create this figure are available.

are no other objects with an L supergiant classification, so we select a range of spectra for comparison to the SpeX observations of V838 Mon, drawing both from late-M and L dwarf spectra and late-M giant and supergiant spectra. In Figure 4, we show the SpeX spectrum of V838 Mon compared to publicly available IR spectra (Cushing et al. 2005; Rayner et al. 2009) of M8V LP 412-31 (similar to its initial optical classification), M8/9III IRAS14303-1042 (as an example lateM giant), and M7I MY Cep (as an example of a late-M supergiant), and L3V 2MASS J00361617+1821104 (possibly similar in surface temperature) from the IRTF Spectral Library. ${ }^{23}$ Each of these spectra share some spectral features with V838 Mon.

The best match for the overall shape of the V838 Mon spectrum is a mid-L dwarf, so we apply $\mathrm{L}$ dwarf classification indices to the IR spectrum of V838 Mon. L dwarfs are classified not only on their spectral type $/ T_{\text {eff }}$, but also their surface gravity (which shows a dependency on age; Burrows et al. 1997). Based on the strength of its $\mathrm{H}_{2} \mathrm{O}$ bands,

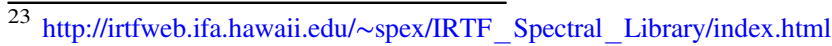


which primarily trace effective temperature, V838 Mon has an IR spectral type of L3. The Allers \& Liu (2013) gravity indices (based on the strength of $\mathrm{K} \mathrm{I}, \mathrm{VO}$, and $\mathrm{FeH}$ ) indicate a very low gravity. $\mathrm{K} \mathrm{I}$ and $\mathrm{FeH}$ are in fact completely absent from the spectrum, consistent with the very low surface gravity L supergiant classification (Evans et al. 2003).

Despite the L3 classification, the detailed spectral features of V838 Mon are not a close match to those of L3 dwarfs, as can be seen in Figure 4. The $\mathrm{TiO}$ bands between 0.8 and 0.9 microns, discussed in Section 4.1, are not typically present in $\mathrm{L}$ dwarf spectra. The very low surface gravity of V838 Mon results in the absence of $\mathrm{FeH}, \mathrm{K}$, and $\mathrm{Na}$ I between 1.1 and $1.3 \mu \mathrm{m}$. Another dramatic difference between L dwarfs and V838 Mon is the presence of AlO A-X absorption bands (first identified on V838 Mon by Bernstein et al. 2003) at 1.226, $1.242,1.646$, and $1.648 \mu \mathrm{m}$. These bands are consistent with oxygen-rich, low temperature environments and have been observed in a handful of AGB stars (Banerjee et al. 2012).

The best match for the detailed $J$-band spectrum of V838 Mon is that of the M8/9II IRAS14303-1042; both have TiO, $\mathrm{VO}$, and $\mathrm{H}_{2} \mathrm{O}$ absorption features, though the specific shapes and strengths of the features are not well matched. The $H$-band spectrum of V838 Mon is dominated by $\mathrm{H}_{2} \mathrm{O}, \mathrm{CO}$, and $\mathrm{OH}$, resulting in detailed features very similar to those of the M7I MY Cep (excepting the AlO absorption bands, present only in V838 Mon). The $K$-band spectra of all three comparison stars are a good overall match to V838 Mon, but the CO bands appear different in both shape and strength, possibly due to the complexities of the dust shell surrounding V838 Mon.

The CO bands in our 2008 spectrum are qualitatively similar to those detected in the Geballe et al. (2007) low-resolution 2006 IR spectra. Geballe et al. (2007) analyzes the CO bands in detail using a high resolution $(R \sim 18000)$ spectra taken within a few months of the low resolution detection; this highresolution spectra revealed distinct velocity components within the $\mathrm{CO}$ spectrum. The main component of the $\mathrm{CO}$ was at photospheric temperatures, but with a radial velocity of $-15 \mathrm{~km} \mathrm{~s}^{-1}$ relative to the stellar radial velocity, indicating the gas was still settling on the surface of the star. Other components of the $\mathrm{CO}$ absorption were associated with the more extended dust shell. In our 2008 spectrum, V838 Mon's CO bands are stronger than those of all the comparison objects, which may indicate that some of the extended dust shell components still contribute to the absorption, but we do not have the velocity resolution to investigate the $\mathrm{CO}$ bands in more detail.

V838 Mon is relatively free of molecular absorption near 1.3 and $2.2 \mu \mathrm{m}$, revealing weak absorption lines. These lines appear qualitatively similar to those of the M7I MY Cep and are likely a feature of supergiant atmospheres. Using the list of IR lines in Arcturus (Rayner et al. 2009), we tentatively identify a Mn I $\lambda 1.2903,1.2980 \mu$ m doublet; an Al I $\lambda 1.3127$, $1.3153 \mu \mathrm{m}$ doublet; and a Si I $\lambda 1.3181 \mu \mathrm{m}$ absorption line in the $1.25-1.32 \mu \mathrm{m}$ spectral region. Near $\sim 2.2 \mu \mathrm{m}$, we tentatively identify Si I at $2.2069 \mu \mathrm{m}$ (not the $\mathrm{Na}$ I doublet seen in the dwarf spectra); a Ti I $\lambda 2.2217,2.2239,2.2280 \mu$ m triplet; and a Ca I $\lambda 2.2614,2.2631,2.2633,2.2657 \mu \mathrm{m}$ quadruplet. Both regions have additional lines of similar strength that overlapped with multiple atomic transitions so they could not be positively identified at this resolution.

\subsection{Estimating $T_{\text {eff }}$ from Spectra}

The optical and IR spectra of V838 Mon, at first glance, seem to indicate different spectral types, and therefore different surface temperatures $\left(T_{\text {eff }}\right)$. Adopting the M7 optical spectral type and extrapolating the supergiant spectral type $/ T_{\text {eff }}$ relation of Levesque et al. (2005) gives a surface temperature of $T_{\text {eff }} \sim 3000 \mathrm{~K}$ (similar to the temperature quoted by Tylenda et al. 2011). There is no supergiant temperature scale for $\mathrm{L}$ dwarfs, but an L3 dwarf spectral type corresponds to $T_{\text {eff }} \sim 1800 \mathrm{~K}$ (Stephens et al. 2009). One possible way of reconciling those two temperatures is an application of a multilayer photosphere (e.g., Lynch et al. 2007), but here we estimate based on the comparison of V838 Mon to other stars.

As discussed in Section 4.1, the optical spectrum for V838 Mon matches an M7 dwarf best because the $\mathrm{TiO}$ bands are strongest on $\mathrm{M} 7$ photospheres. The fraction of $\mathrm{TiO}$ contained in molecules (versus Ti I) increases with decreasing temperature, hitting a maximum (at $\mathrm{M}$ dwarf atmospheric pressures) at $T_{\text {eff }} \sim 2000 \mathrm{~K}$ (Lodders \& Fegley 2006). At temperatures cooler than $T_{\text {eff }} \lesssim 2400 \mathrm{~K}, \mathrm{TiO}$ begins to condense onto dust grains (e.g., perovskite; Burrows \& Sharp 1999), so TiO disappears in M7 and later dwarfs. The formation of dust grains is more sensitive to atmospheric pressure than the formation of $\mathrm{TiO}$, so at low surface gravity, a low temperature atmosphere will continue to have strong $\mathrm{TiO}$ bands at $T_{\text {eff }} \lesssim 2400 \mathrm{~K}$.

The remaining indicators of cool temperatures in optical spectra (e.g., the transition from late-M to $\mathrm{L}$ dwarfs) are pressure sensitive: gravity broadening of the $\mathrm{K}$ I doublet, the strengthening of FeH. Additionally, the IR spectrum of V838 Mon appears cooler than the M7I MY Cep due to stronger TiO, $\mathrm{VO}$, and $\mathrm{H}_{2} \mathrm{O}$ bands. Therefore, the spectrum of V838 Mon may be both very cooler and very low gravity; a prototype for $\mathrm{L}$ supergiants in the optical. M giants are $T \sim 200-400 \mathrm{~K}$ warmer than dwarfs of the same optical type, and if this trend continues, the surface temperature of V838 Mon is likely to be $T_{\text {eff }} \sim 2000-2200 \mathrm{~K}$. This range of temperatures is also consistent with the formation of strong $\mathrm{TiO}$ bands without significant depletion of $\mathrm{TiO}$ onto dust.

\section{INFRARED SEDS}

In this section, we fit our visible and IR data with a two component blackbody curve. We consider the physical interpretation of this fit in context of the Lynch et al. (2004), henceforth, L04, model of V838 Mon's post outburst evolution. Our goal is to constrain the evolution of the ejecta with our new epoch of data. The L04 model contains three components.

1. The central star's photospheric emission, which is modeled as an inner blackbody source with a homogeneous outer, cooler absorbing layer. The central star is described by a radius, $R_{p}$, and a blackbody temperature, $T_{\mathrm{BB}}^{p}$.

2. A warm shell, which is modeled with several parameters, including an expanding radius, $R_{s}$, and a vibrational/ blackbody emission temperature, $T_{\mathrm{BB}}^{s}$.

3. A cold, outer shell, modeled in a similar fashion as the warm, expanding shell, but with an emission temperature set to zero. Here, the quantity of greatest significance is the absorber column (resulting in an effective emissivity, $\epsilon$, of the system).

We also considered the Lynch et al. (2007), henceforth, L07, model of V838 Mon's ejecta, which contain five components (a 
Table 2

Best Fit Parameters for Two Component Model

\begin{tabular}{|c|c|c|c|c|c|c|}
\hline & Year & $R_{p}(\mathrm{AU})$ & $T_{\mathrm{BB}}^{p}(\mathrm{~K})$ & $R_{s}(\mathrm{AU})$ & $T_{\mathrm{BB}}^{s}(\mathrm{~K})$ & $\epsilon$ \\
\hline L04 & 2003 & 5.6 & 2100 & 28 & 750 & 0.67 \\
\hline L07 & 2005 & 4.2 & 2100 & 129.25 & 375 & $\ldots$ \\
\hline Predictions based on L04 model & 2009 & 4.2 (adopting L7 value) & 2100 & 230.5 & 283 & 0.67 \\
\hline Best fit to observed data (current work) & 2009 & $4.3 \pm 2$ & $2370 \pm 400$ & $263 \pm 10$ & $285 \pm 2$ & $0.65 \pm .2$ \\
\hline
\end{tabular}

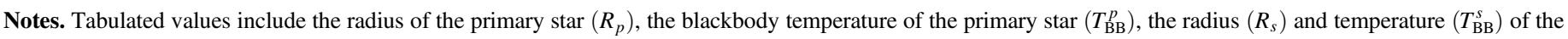

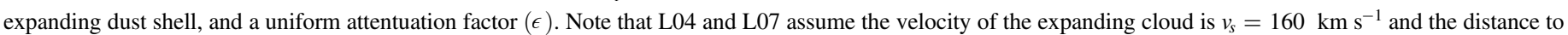
V838 Mon is $\mathrm{D}_{\mathrm{V} 838}$ Mon $\sim 6 \mathrm{kpc}$.

central star, two photospheric shells that surround the central star, and two much cooler and more distant expanding shells of gas). This model is essentially the same as the L04 model, except the two photospheric layers are considered separately from the central star, and are each assumed to be homogeneous and characterized by a single equilibrium temperature and column amounts for the molecular species. While the added components are physically reasonable and lead to refined predictions for $T_{\mathrm{BB}}^{p}$, the added complexity beyond this revision did not help us build additional insight from our two component blackbody fit. Therefore, our remaining discussion will focus primarily on comparisons with the L04 model.

Best fits from L04 and L07 can be found in Table 2. We note that the generally accepted values for the velocity of the expanding cloud in 2003 were within $100-400 \mathrm{~km} \mathrm{~s}^{-1}$ (see L04 and references therein). We assume the velocity of the expanding cloud is $v_{s}=160 \mathrm{~km} \mathrm{~s}^{-1}$ and the distance to V838 Mon is $D_{\mathrm{V} 838 \mathrm{Mon}}=6 \mathrm{kpc}$ (Sparks et al. 2008) to generate model predictions. In 2009 January, L04 predicts: $R_{p} \sim 4.2$ AU (no evolution from 2005); $T_{B B}^{p} \sim 2100 \mathrm{~K}$ (no evolution from 2005); $R_{S} \sim 230.5 \mathrm{AU}$ (time elapse 6 year 10.5 months, expansion rate $33.75 \mathrm{AU} \mathrm{yr}^{-1}$ ); and $T_{B B}^{s} \sim 283 \mathrm{~K}$ (temperature of cloud in $2003 \times 0.38)$.

We fit our 2009 SED data, consisting of both de-reddened optical and IR data, with a two component blackbody curve (Figure 5). Our simultaneous fit yielded for the primary star $T_{p}=2370 \pm 400 \mathrm{~K}$ and $R_{p}=4.3 \pm .2 \mathrm{AU}$ and $T_{s}=285 \pm 2$ $\mathrm{K}, R_{s}=263 \pm 10 \mathrm{AU}$, and veiling $=35 \% \pm 20$ for the warm expanding dust envelope. We note that the $T_{\text {eff }}$ identified in section Section 4 using spectral analysis $\left(T_{\text {eff }} \sim 2000-2200 \mathrm{~K}\right)$ falls within the $3 \sigma$ errors on our best fit for the temperature of the primary, $T_{p}=2370 \pm 400 \mathrm{~K}$.

Our results are consistent with the predictions from the L04 model. We find only a slightly larger radius for the expanding dust shell $\left(R_{s} \sim 263 \pm 10 \mathrm{AU}\right)$ than is predicted $\left(R_{s} \sim 230.5\right.$ AU) using an expansion rate of $v_{s}=160 \mathrm{~km} \mathrm{~s}^{-1}$. Moreover, the blackbody temperature we determine for this shell $\left(T_{\mathrm{BB}}^{s}\right.$ $\sim 285 \pm 2 \mathrm{~K})$ is consistent with that predicted from the L04 model.

\section{DISCUSSION AND CONCLUSIONS}

In this section, we discuss and present our conclusions regarding V838 Mon's spectral type, expanding outburst ejecta, and ongoing $\mathrm{H} \alpha$ emission. We also comment on the lack of new dust formation (relative to 2008 observations) and place this in the context of the evolution of the system as a whole.

In Section 4, we concluded that the IR spectral slope of V838 Mon is best matched to an L3 supergiant. While the optical data is best matched by a spectral classification of M7 (as matched to a dwarf template), this classification does not take into account the effects of low gravity on the persistence of various spectral features. Significantly, the temperatures at which $\mathrm{TiO}$ can remain coherent in supergiant photospheres could be as high as 2000-2200 K. Given the complete lack of L supergiant data to type against within the optical regime, we conclude that V838 Mon could plausibly be catagorized as an L supergiant in both the IR and optical regimes. In this regard, we speculate that V838 Mon could be a prototype for what an L supergiant optical spectra should look like.

In Section 5, we used our 2009 optical to mid-IR observations as a third epoch of data to test the Lynch et al. (2004) model of the expanding ejecta envelope. Our data indicate the dust shell has continued to cool at a similar rate as suggested by the Lynch et al. (2004) model. If the shell was ejected at a velocity of $160 \mathrm{~km} \mathrm{~s}^{-1}$, the 2009 IR data is consistent with a $285 \pm 2 \mathrm{~K}$ shell of material which has expanded to a radius of $263 \pm 10 \mathrm{AU}$ and is attenuated by $\sim 35 \% \pm 20$ by an outer cooler shell. In this best fit to the model, the radius of the L3 primary is described by $R_{p} \sim 4.3 \pm 0.2 \mathrm{AU}$ and $T_{p} \sim 2370 \pm 400 \mathrm{~K}$. We note that the $T_{\text {eff }}$ identified in Section 4 using spectral analysis $\left(T_{\text {eff }} \sim 2000-2200 \mathrm{~K}\right)$ falls within the $3 \sigma$ errors on our best fit for the temperature of the primary, $T_{p}=2370 \pm 400 \mathrm{~K}$.

These results are consistent with the recent IR interferometric analysis by Chesneau et al. (2014). Using the Very Large Telescope Interferometer between 2011 October and 2012 February, and adopting a distance to V838 Mon of $D=6.1 \pm 0.6 \mathrm{kpc}$, Chesneau et al. (2014) deduce that there is a dust shell distributed from about 130 to 300 AU around V838 Mon. They note that the dust is distributed in a flattened structure, which could be interpreted as a relic of the genesis event or could be influenced by the embedded B3V companion. They also note that as of 2014, the radius of V838 Mon's photosphere has decreased by about $40 \%$ from the radius determined after the outbursting events to $3.5 \pm 0.6 \mathrm{AU}$. We note that the radius of the primary that we determined $(4.3 \pm 0.2 \mathrm{AU})$ is within the error bars of the radius determined by Chesneau et al. (2014).

We consider here whether the dust is newly formed material or persisting matter that has expanded to a larger spatial distribution. Wisniewski et al. (2008) previously found substantial dust production in the 2006-2007 epoch, which was reflected in an IR excess at the $10-80 \mu \mathrm{m}$ wavelength range. We repeat their analysis on our 2008-2009 IR photometry. In Figure 6, we plot our new results together with the data presented in Wisniewski et al. (2008). As is evident, our 2008-2009 IR photometry shows no appreciable change from the 2006-2007 epoch. We conclude that there has been no substantive change in the dust production or composition. 


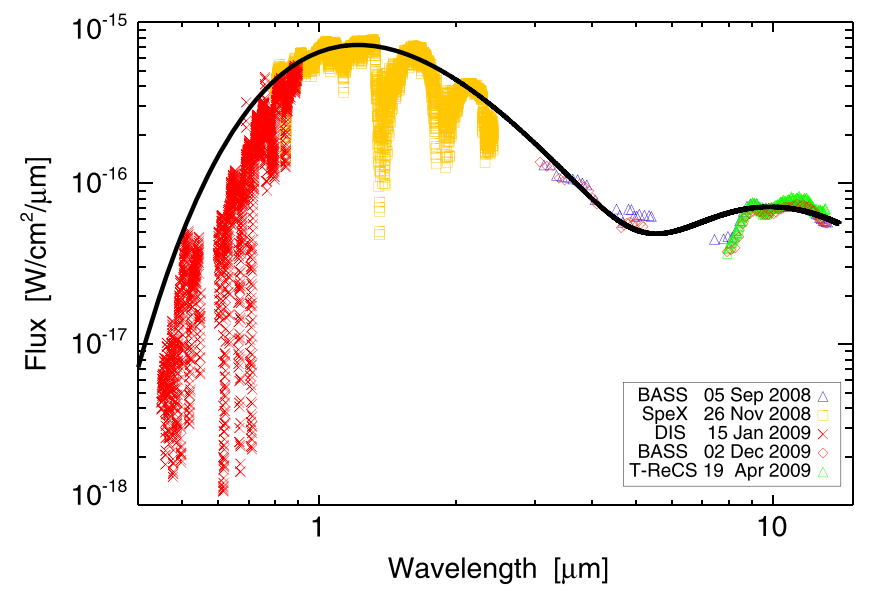

Figure 5. We fit a two component blackbody model to V838 Mon, including a $2370 \mathrm{~K}$ component from the primary star and a $285 \mathrm{~K}$ component from the warm expanding envelope. These data provide observational support for the Lynch et al. (2004) model.

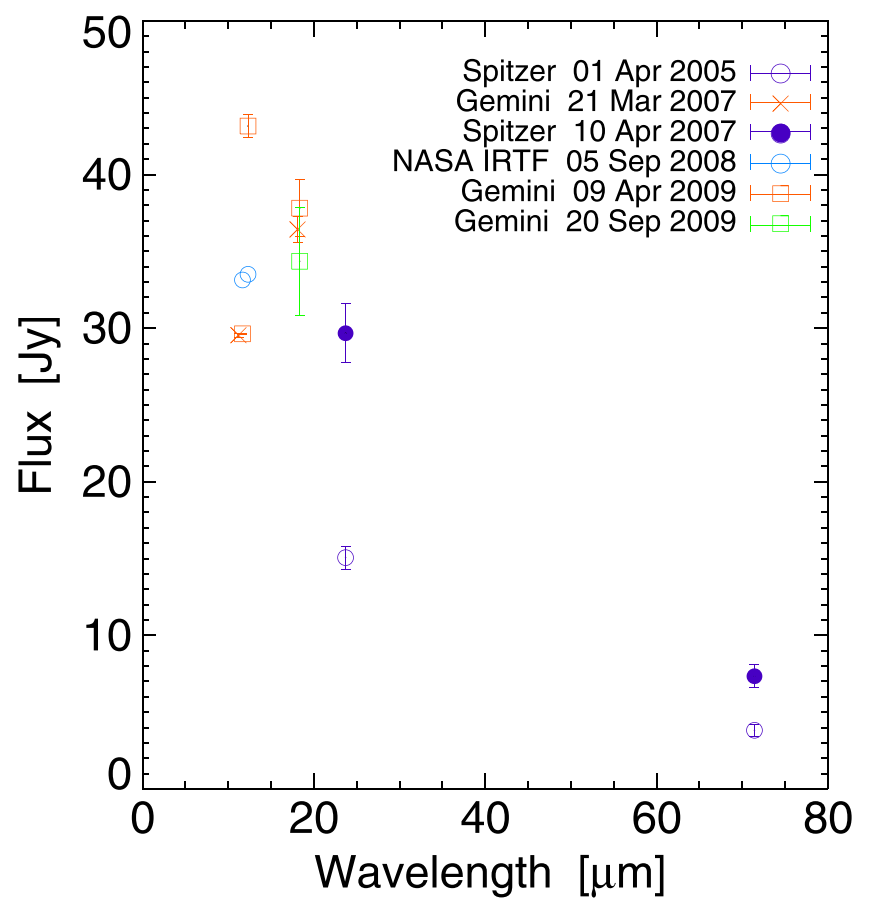

Figure 6. Our 2008-2009 IR photometry shows no appreciable change from the 2006-2007 epoch (Wisniewski et al. 2008). We conclude there has been no substantive change in the dust production or composition. The data used to create this figure are available.

In Section 3, we showed that the level of $\mathrm{H} \alpha$ activity has declined since the line's brief return to significant emission in 2006. While $\mathrm{H} \alpha$ emission is not distinguishable in low resolution spectra obtained during 2008-2009, all highresolution $(\mathrm{R} \sim 31500)$ spectra obtained between $2008-2012$ do show the ongoing presence of a small amount of $\mathrm{H} \alpha$ emission. We suggest two possible origins for the observed weak $\mathrm{H} \alpha$ emission. The emission could originate from magnetic activity in the primary star, which is an expected phenomenon (Soker \& Tylenda 2007) if the 2002 outburst was caused by a stellar merger event (Tylenda \& Soker 2006).
Antonini et al. (2010) found highly variable X-ray emission associated with V838 Mon: in 2008, strong X-ray emission was detected with XMM Newton, but in 2009 and 2010 no such emission was found using Chandra. From this, Antonini et al. (2010) suggest that the magnetic activity itself might be highly variable. We do not detect significant $\mathrm{H} \alpha$ variability in our high resolution spectra that supports the purported variable magnetic activity; however, we cannot rule out that this is due to the sparse, non-contemporaneous sampling of the optical and Xray data. Alternatively, the emission may simply be caused by the continued excitation of $\mathrm{Hi}$ in the expanding shell of gas and dust by the $\mathrm{B} 3 \mathrm{~V}$ binary companion, which would indicate that this shell is not completely optically thick.

To ultimately disentangle which scenario is generating the $\mathrm{H} \alpha$ emission, a simultaneous X-ray and observational campaign is needed. As Antonini et al. (2010) note, it is implausible to generate high X-ray luminosities from the simple interactions of infalling matter with the matter above the stellar photosphere unless extreme accretion rates and infall velocities are involved. Thus, catching V838 Mon in the act of producing strong X-ray emission would be a strong indicator that the stellar merger is the ultimate source of any ongoing $\mathrm{H} \alpha$ activity. Even if no X-ray emission is detected, it is certainly worthwhile to continue to monitor the $\mathrm{H} \alpha$ activity and search for the reappearance of the B3V companion. Further evolution (or lack thereof) will put strong contraints on both the material surrounding V838 Mon and the genesis event that fueled the outbursts.

This work is supported at The Aerospace Corporation by the Independent Research and Development program. S.R.L. acknowledges support from the Michigan Society of Fellows. M.M.K. acknowledges generous support from the Hubble Fellowship and Carnegie-Princeton Fellowship.

\section{REFERENCES}

Afşar, M., \& Bond, H. E. 2007, AJ, 133, 387

Allers, K. N., \& Liu, M. C. 2013, ApJ, 772, 79

Antonini, F., Montez, R. Jr., Kastner, J. H., et al. 2010, ApJ, 717, 795

Banerjee, D. P. K., Varricatt, W. P., Mathew, B., Launila, O., \& Ashok, N. M. 2012, ApJL, 753, L20

Barsukova, E. A., Goranskij, V. P., Metlova, N. V., Kroll, P., \& Valeev, A. F. 2010, in XXVIth IAP Annual Colloquium, Progenitors and Environments of Stellar Explosions (Melville, NY: AIP), 43

Bernstein, L. S., Rudy, R. J., Lynch, D. K., et al. 2003, IAU Circ., 8082, 2

Bochanski, J. J., West, A. A., Hawley, S. L., \& Covey, K. R. 2007, AJ, 133,531

Bond, H. E. 2009, AAS Meeting Abstracts, 214, 430.06

Bond, H. E., Bedin, L. R., Bonanos, A. Z., et al. 2009, ApJL, 695, L154

Bond, H. E., et al. 2003, Natur, 422, 405

Brown, N. J., Waagen, E. O., Scovil, C., et al. 2002, IAU Circ., 7785, 1

Burrows, A., et al. 1997, ApJ, 491, 856

Burrows, A., \& Sharp, C. M. 1999, ApJ, 512, 843

Chesneau, O., Millour, F., DeMarco, O., et al. 2014, A\&A, 569, 3

Covey, K. R., et al. 2007, AJ, 134, 2398

Cushing, M. C., Rayner, J. T., \& Vacca, W. D. 2005, ApJ, 623, 1115

Cushing, M. C., Vacca, W. D., \& Rayner, J. T. 2004, PASP, 116, 362

Evans, A., Geballe, T. R., Rushton, M. T., et al. 2003, MNRAS, 343, 1054

Geballe, T. R., Rushton, M. T., Eyres, S. P. S., et al. 2007, A\&A, 467, 269

Goranskii, V. P., Kusakin, A. V., Metlova, N. V., et al. 2002, AstL, 28, 691

Goranskij, V., Zharova, A., Barsukova, E., Fabrika, S., \& Valeev, A. 2008, arXiv:0810.1887

Hackwell, J. A., Warren, D. W., Chatelain, M. A., Dotan, Y., \& Li, P. H. 1990, Proc. SPIE, 1235, 171

Kasliwal, M. M., et al. 2011, ApJ, 730, 134

Kochanek, C. S. 2011, ApJ, 741, 37

Kulkarni, S. R., et al. 2007, Natur, 447, 458 
Lawlor, T. M. 2005, MNRAS, 361, 695

Levesque, E. M., Massey, P., Olsen, K. A. G., et al. 2005, ApJ, 628, 973

Lodders, K., \& Fegley, B. Jr. 2006, in Chemistry of Low Mass Substellar Objects, ed. J. W. Mason (Berlin: Springer), 1

Lynch, D. K., et al. 2007, in ASP Conf. Ser. 363, The Nature of V838 Mon and its Light Echo, ed. R. L. M. Corradi, \& U. Munari (San Francisco, CA: ASP), 39

Lynch, D. K., et al. 2004, ApJ, 607, 460

Martini, P., Wagner, R. M., Tomaney, A., et al. 1999, AJ, 118, 1034

Mould, J., et al. 1990, ApJL, 353, L35

Munari, U., et al. 2005, A\&A, 434, 1107

Munari, U., Navasardyan, H., \& Villanova, S. 2007, in ASP Conf. Ser. 363, The Nature of V838 Mon and its Light Echo, ed. R. L. M. Corradi, \& U. Munari (San Francisco, CA: ASP), 13

Pickles, A. J. 1998, PASP, 110, 863

Prieto, J. L., et al. 2008, ApJL, 681, L9

Rayner, J. T., Cushing, M. C., \& Vacca, W. D. 2009, ApJS, 185, 289

Rayner, J. T., Toomey, D. W., Onaka, P. M., et al. 2003, PASP, 115, 362
Retter, A., Zhang, B., Siess, L., \& Levinson, A. 2006, MNRAS, 370, 1573

Rushton, M. T., et al. 2005, MNRAS, 360, 1281

Schiavon, R. P., Barbuy, B., Rossi, S. C. F., \& Milone, A. 1997, ApJL, 479,902

Soker, N., \& Tylenda, R. 2007, MNRAS, 375, 909

Sparks, W. B., et al. 2008, AJ, 135, 605

Stephens, D. C., et al. 2009, ApJ, 702, 154

Thompson, T. A., Prieto, J. L., Stanek, K. Z., et al. 2009, ApJ, 705, 1364

Tylenda, R., \& Kamiński, T. 2012, A\&A, 548, A23

Tylenda, R., Kamiński, T., Schmidt, M., Kurtev, R., \& Tomov, T. 2011, A\&A, 532, A138

Tylenda, R., \& Soker, N. 2006, A\&A, 451, 223

Vacca, W. D., Cushing, M. C., \& Rayner, J. T. 2003, PASP, 115, 389

Wang, S.-i., et al. 2003, Proc. SPIE, 4841, 1145

Wisniewski, J. P., Bjorkman, K. S., \& Magalhães, A. M. 2003, ApJL, 598, L43

Wisniewski, J. P., Clampin, M., Bjorkman, K. S., \& Barry, R. K. 2008, ApJL, 683, L171

Wisniewski, J. P., Morrison, N. D., Bjorkman, K. S., et al. 2003, ApJ, 588, 486 